\title{
EL PAPEL DE LA EDUCACIÓN GENERAL EN LOS ESTUDIOS UNIVERSITARIOS CONTEMPORÁNEOS \\ Ana Margarita Haché*
}

RESUMEN: Se describe cuál podría ser el impacto de los Estudios Generales en la educación universitaria, en particular, en República Dominicana; asimismo, se argumenta por qué sería deseable la inclusión de dichos estudios en el currículo universitario.

soes

ABSTRACT: In this article, we explore the potential impact of General Studies in universities, particularly in the Dominican Republic, and the advantages of such inclusion in the university curriculum.

PALABRAS ClAVE: Educación general, Estudios Generales, Universidad, especialización, República Dominicana.

KEY WORDS: General education, General Studies, University, specialization, Dominican Republic.

RECEPCIÓN: 23 de noviembre de 2010. APROBACIÓN: 7 de diciembre de 2010.

* Departamento de Humanidades, Pontificia Universidad Católica Madre y Maestra. 
CITAM Derechos Reservados.

La reproducción total o parcial de este artículo se podrá hacer si el ITAM otorga la autorización previamente por escrito. 


\title{
EL PAPEL DE LA \\ EDUCACIÓN GENERAL EN LOS ESTUDIOS UNIVERSITARIOS CONTEMPORÁNEOS*
}

\begin{abstract}
Agradecemos a los organizadores de este II Simposio Internacional de Estudios Generales la invitación que nos hicieran para participar en este panel. Nos honra sobremanera poder compartir esta mesa con tan distinguidas autoridades académicas e intelectuales. Estamos seguros de que todos concordarán con nuestro deseo de dedicar esta exposición al Lic. Francisco Polanco, quien fuera un abanderado de los Estudios Generales en la Madre y Maestra.

Es conveniente explicitar la pregunta que subyace al título de este panel: ¿Cuál es la contribución que los Estudios Generales pueden aportar a las universidades en este siglo que recién inicia? Las respuestas que pudieran suscitarse serían de vital importancia para definir la pertinencia de los Estudios Generales en la educación superior, dentro de la cultura contemporánea, y podrían dar luz a una reforma curricular que se hace necesaria en estos momentos en las instituciones de educación superior dominicanas.

Abordar la temática de este panel requiere despejar, entre otras, tres incógnitas: qué se entiende por educación general, cuál es la educación universitaria de hoy en nuestro país, y cómo se define y caracteriza esta época del siglo XXI. Estos tres aspectos sirvieron de base a la presentación que haremos a continuación. Ellos se entrelazan y se suponen en

* Segundo Simposio Internacional de Estudios Generales. Pontificia Universidad Católica Madre y Maestra, 27, 28 y 29 de octubre de 2010, Santiago, República Dominicana.
\end{abstract}


un discurso que pretende resaltar los beneficios de la educación general en tiempos de globalización en la República Dominicana.

Definir la educación general no es tarea fácil, como lo constata Waldemiro Vélez en su trabajo Trayectoria histórica de la Educación General en los currículos universitarios en los Estados Unidos: "Todavía en el 1977, la Carnegie Foundation for the Advancement of Teaching, en un trabajo titulado Missions of the College Curriculum, afirmaba que ningún concepto curricular es tan fundamental para el quehacer de las universidades norteamericanas como el de la educación general; y ninguno se encuentra más lejos que éste, de un acuerdo general sobre su significado". ${ }^{1}$

Dada la pluralidad de sentidos de los Estudios Generales, repasaremos diversas definiciones para extraer de ellas características de la educación general que pueden fortalecer a los estudios universitarios en nuestro país. En primer lugar, veamos la definición que ofreció Miller, en 1988, al afirmar que la educación general es un programa comprensivo, elaborado conscientemente, para desarrollar en el estudiante la actitud de búsqueda, las destrezas para solucionar problemas, los valores individuales y comunitarios asociados a una sociedad democrática, y el conocimiento que se necesita para aplicar esas actitudes y valores que el estudiante mantiene en el proceso de aprendizaje a lo largo de su vida, participando activamente y comprometido con los cambios que deben darse en todo proceso democrático.

A partir de esta conceptualiuzación, una primera contribución de la educación general a los estudios universitarios de hoy, se centra en lo que Miller denomina "actitud de búsqueda". En el mundo occidental, esta visión tuvo sus raíces fundacionales en Grecia. Evidentemente, la criticidad y la reflexividad son características inherentes a nuestra humanidad; sin embargo, no siempre han ido de la mano de la educación. Pablo Freire ha criticado esta falta, abogando por una educación liberadora. Se trata de una pedagogía donde la pregunta ocupa un lugar privilegiado.

${ }^{1}$ W. Vélez, "Trayectoria histórica de la Educación General en los currículos universitarios en los Estados Unidos", Ponencia presentada en el Seminario de Educación General de la Facultad de Estudios Generales, 21 de septiembre de 2007. [http://umbral.uprrp.edu/materiales/conferencias-77]. 
¿En que consistiría una educación de este tipo? Según Martha Nussbaum, la primera habilidad para cultivar la humanidad en el mundo actual es "la habilidad para un examen crítico de uno mismo y de las propias tradiciones, que nos permita experimentar lo que podríamos llamar una vida examinada". ${ }^{2}$ Para Sócrates, una vida sin examen interior no vale la pena. "En otras palabras -sigue diciendo Nussbaum-, esta vida de cuestionamiento no es sólo algo útil; es componente indispensable de una vida con sentido para cualquier persona y cualquier ciudadano". ${ }^{4}$

¿Por qué se hace imperativa esta característica de la educación general? ¿A qué se debe que le atribuyamos un papel preponderante a la criticidad y la reflexividad en esta etapa de la contemporaneidad? Para Gimeno Sacristán, la condición en la que nos encontramos hoy no es otra que la globalización. ${ }^{5}$ Este fenómeno, fuertemente ligado al neoliberalismo y a las nuevas tecnologías de la comunicación y la información, tiene, como afirma Carlos Fuentes, dos caras: una buena, que abarca el avance técnico y científico más veloz de toda la historia; y otra menos atractiva y perversa: "la velocidad misma del desarrollo tecnológico deja atrás, quizás para siempre, a países incapaces de mantener el paso". ${ }^{6}$

En este sentido, la globalización crea desigualdad y desequilibrios a nivel mundial y a lo interno de cada país. Para valorar adecuadamente el momento en que vivimos y sus consecuencias, se requiere, hoy más que nunca, de seres humanos reflexivos y críticos que puedan ejercer con responsabilidad su ciudadanía. Además, en un mundo donde el relativismo y el consumo parecen haberse apoderado de él, el autoexamen se convierte en una poderosa cualidad que impide el adocenamiento y realza nuestra condición humana. Una educación que promueva en los sujetos la reflexividad propia de la educación general conlleva el germen de la libertad, al permitirles adueñarse de su historia y de las consecuencias de sus acciones, lo que es de vital importancia para una cultura comprometida con la justicia, como diría Nussbaum.

${ }^{2} \mathrm{M}$. Nussbaum, El cultivo de la humanidad. Una defensa clásica de la reforma en la educación liberal, 2001, Barcelona, Ediciones Paidós Ibérica, p. 28.

${ }^{3} \mathrm{Pl}$. Ap., 38a.

${ }^{4}$ Nussbaum, op. cit., p. 42.

${ }^{5}$ J. Gimeno Sacristán, La educación que aún es posible, 2005, Madrid, Ediciones Morata, p. 15.

${ }^{6}$ C. Fuentes, En esto creo, 2002, México, Seix Barral, p. 88-9. 
Por otro lado, el desarrollo del pensamiento crítico en el estudiantado universitario ayudaría a valorar esta etapa de la modernidad, que prioriza la ciencia y la tecnología en detrimento de las humanidades. En efecto, el currículo de la educación general permite analizar el impacto de la tecnología en la sociedad y puede, en palabras de Waldemiro Vélez, "comenzar por desmitificar la ciencia y sus aplicaciones industriales (la tecnología)". 7 En este sentido, Edgar Morin recomienda que "la educación debe favorecer la aptitud natural del pensamiento para plantear y resolver problemas y, correlativamente, estimular el pleno empleo de la inteligencia general, cuyo desarrollo está relacionado con la duda, germen de toda actividad crítica". ${ }^{8}$

El desarrollo de los valores individuales y comunitarios, asociados a una sociedad democrática y el conocimiento que se necesita para aplicarlos, participando activa y comprometidamente con los cambios que deben darse en todo proceso democrático, constituye otro de los beneficios que la educación general aporta a los estudios universitarios de este siglo. En mayor o menor medida, la mayoría de los países del mundo viven en democracia. Aunque exista una pérdida de la confianza que la gente otorgaba a los políticos y a los procedimientos formalistas de la democracia, todavía se tiene fe en aquellos procesos democráticos que realmente auspician y permiten la participación ciudadana a todos los niveles.

Vivir en democracia supone reconocer "lo diferente y las diferencias". Aun dentro de nuestro país, la diversidad cultural nos lleva a superar el viejo concepto de una identidad monolítica, para entonces, empezar a hablar de "identidades dominicanas". Para Barone y Mella, "en una sociedad de masas, cultivar la pluralidad significa reaccionar a la homogenización de los seres humanos". ${ }^{9}$ La educación general se

${ }^{7}$ W. Vélez, "El currículo de educación general en el Siglo XXI", Ponencia presentada en el XII Encuentro Nacional de Educación y Pensamiento, Ponce, Puerto Rico, 29-31 de marzo de 2000, p. 4. [http://umbral.uprrp.edu/materiales/conferencias-33].

${ }^{8}$ E. Morin, La cabeza bien puesta. Repensar la reforma; reformar el pensamiento, 2002, Buenos Aires, Nueva visión, p. 24.

${ }^{9}$ S. Barone y Mella, P., "Acción educativa y desarrollo humano en la universidad de hoy", Revista Iberoamericana de Educación, 31, Madrid, Organización de Estados Iberoamericanos para la Educación, la Ciencia y la Cultura, 2003, p. 206. 
identifica con lo que estos autores llaman, siguiendo a Hanna Arendt, "la acción educativa". Dicha acción actualiza una dimensión de la existencia que valoriza la multiplicidad de los discursos humanos. Esta perspectiva, presente en los Estudios Generales, resulta fundamental para una sociedad donde las desigualdades socioeconómicas se convierten en barreras para adquirir el estatus de ciudadano y para un país que, dentro de una misma isla, debe convivir con otra nación. Por eso, la presencia de asignaturas sobre la realidad dominicana y la cultura haitiana deberían tener garantizado un espacio en nuestros estudios universitarios.

Educar para una ciudadanía responsable, como aboga la educación general, implica lo que Edgar Morin llama “enseñar la comprensión”. Este autor afirma: "El problema de la comprensión se ha vuelto crucial para los humanos. Y, por esta razón, debe ser una de las finalidades de la educación del futuro". La gran paradoja de la globalización estriba en que, la interconectividad que la sociedad del Internet nos posibilita, no trae aparejada una adecuada comprensión humana. Ésta, para Morin, "implica un conocimiento de sujeto a sujeto [...] Siempre intersubjetiva, la comprensión humana necesita apertura, simpatía y generosidad", ${ }^{10}$ Recordemos que, para Touraine, "sólo podremos vivir juntos con nuestras diferencias si nos reconocemos mutuamente como sujetos". ${ }^{11}$ Ahí radica la misión espiritual de la educación para Morin: enseñar la comprensión entre las personas como condición y garantía de la solidaridad intelectual y moral de la humanidad. Esta tarea muy bien pueden hacerla posibles los principios que enarbola la educación general.

En efecto, los Estudios Generales pueden contribuir a una transformación del currículo universitario, incorporando una variedad de enfoques sobre la diversidad humana, sobre todo en una época de creciente internacionalización y de multiculturalidad, aunque también signada por la individualidad y, paradójicamente, por la masificación. El enfoque humanista de la educación general nos permite entender, según Nussbaum,

${ }^{10}$ E. Morin, Los siete saberes necesarios para la educación del futuro, 2000, Bogotá, UNESCO, p. 69.

${ }^{11}$ A. Touraine, ¿Podremos vivir juntos? Iguales y diferentes, 1997, México, FCE, p. 166. 
cómo es que las necesidades y objetivos comunes pueden darse de distintas formas en otras circunstancias y lugares.

Otra definición de la educación general que conviene examinar, para valorar sus aportaciones al currículo universitario de hoy, es la expuesta por Kanter, Gamson y London en 1997; plantean que la educación general es el conjunto organizado de actividades designado para promover no solamente el pensamiento crítico y la responsabilidad personal y social, sino también las destrezas de escritura y el conocimiento de las artes liberales.

La educación general adquiere vigencia y significación hoy en los estudios universitarios dominicanos al promover la competencia comunicativa escrita. Dentro de esta competencia, distinguiremos la narración. Aristóteles decía que toda historia bien contada enseña aspectos universales de la condición humana. "Gracias a la narración examinamos la vida, la analizamos en sentido socrático". ${ }^{12}$ Una educación que fortalece la capacidad narrativa del estudiantado le permite una mayor y mejor comprensión de sí mismos, puesto que a través de la trama narrativa, se revela la persona con sus circunstancias. Esta práctica curricular contribuye a fortalecer la identidad de los actores educativos, sobre todo, en una época de la modernidad que se caracteriza por lo que Touraine describe como la fragmentación del sujeto.

Por otro lado, la práctica de la narración cobra sentido, también, en la educación general y es una valiosa herramienta para el cultivo de la humanidad, según lo que Martha Nussbaum denomina imaginación narrativa. "Esto significa la capacidad de pensar: cómo sería estar en el lugar de la otra persona, ser un lector inteligente de la historia de esa persona y comprender las emociones, deseos y anhelos que alguien así pueda experimentar". Esta perspectiva combina la identificación del otro pero cargando con nuestras creencias y juicios. La lectura de una variedad de obras literarias, propia de los estudios generales, proporcionaría al estudiantado esta capacidad que Nussbaum considera como "esencial para cualquier juicio responsable, puesto que no sabremos lo que estamos

${ }^{12}$ T. D. Moratalla, y Mella, P., "Notas para pensar la educación en términos narrativos", Cuaderno de Pedagogía Universitaria, 10, (5), Santiago, Pontificia Universidad Católica Madre y Maestra, 2008, p. 7. 
juzgando hasta no ver el significado de una acción según la intención de la persona que la realiza, ni entenderemos el significado de un discurso mientras no conozcamos la importancia de lo que expresa en el contexto de la historia y el mundo de esa persona". ${ }^{13}$

Otro enfoque que resulta significativo en el desarrollo de las destrezas de escritura, y que representa una de las posibilidades de los Estudios Generales, es la "alfabetización académica". Con este concepto nos referimos al proceso de adquisición de un conjunto de conocimientos lingüísticos y de estrategias cognitivas necesarias para interpretar y producir textos que se utilizan en contextos de estudio. Esta visión pone de manifiesto que el aprendizaje de la lectura y la escritura no terminan con los estudios secundarios; por el contrario, se continúan a lo largo de la vida.

En efecto, los modos de leer y escribir no son iguales en todos los ámbitos del saber. Cada disciplina posee una forma de construir el conocimiento y unos procedimientos para acceder a él que son privativos de ella y su comunidad discursiva; por lo tanto, para aprehender las conceptualizaciones y las metodologías de una determinada área del saber, hay que aprender sus formas de leer y escribir. Así, la escritura se visualiza como una herramienta epistémica con el fin de elaborar el conocimiento y organizarlo. En la organización curricular, este enfoque tiene consecuencias particulares en la forma en que se desarrolla la acción educativa: es desde y en las disciplinas, que se enseña a leer y a escribir al estudiantado.

Asumir la alfabetización académica implica, según Paula Carlino, "aprender a escribir para cada asignatura universitaria, lo que requiere de enseñanza explícita por parte del profesorado especializado y de acciones institucionales para que los docentes puedan llevar a cabo esta práctica educativa". ${ }^{14}$ De este modo, la enseñanza de la lectura y escritura, en otro momento sólo situada en las clases de Español, pasa a ser responsabilidad de las asignaturas que conforman el currículo universitario

${ }^{13}$ Op. cit., p. 30.

${ }^{14}$ P. Carlino, "Prácticas y representaciones de la escritura en la universidad: los casos de Australia, Canadá, Estados Unidos y Argentina", Cuaderno de Pedagogía Universitaria, 12 (6), Santiago, Pontificia Universidad Católica Madre y Maestra, 2009, p. 12. 
como metodologías dependientes de los modos de pensamiento de cada cultura disciplinar. Así, las prácticas de lectura y escritura están fuertemente ligadas a la epistemología de cada área del saber. Lo novedoso de esta perspectiva es que la universidad asume una responsabilidad institucional, para convertir en objeto de enseñanza las prácticas discursivas con las que las disciplinas elaboran el conocimiento. Este compromiso para verdaderamente enseñar a leer y escribir en la academia requiere del consenso del profesorado de todas las asignaturas y de la misma institución de educación superior. Este innovador enfoque tiene cabida en la educación general, cuyo "carácter distintivo se encamina al examen de los fundamentos y procesos de la producción del conocimiento mismo en el contexto de la formación integral del estudiante como sujeto situado histórica y culturalmente". ${ }^{15}$

El conocimiento de las artes liberales determina una parte el contenido curricular de los Estudios Generales. Como apunta Prieto, la tricotomía de Humanidades, Ciencias Naturales y Ciencias Sociales ha mostrado ser útil a través de décadas. ${ }^{16}$ Se trata de una visión integrada de la naturaleza del ser humano, su cultura y su entorno físico y social. Todo esto nos llevaría al estudio de la condición humana que, según Morin, no sólo proviene de las percepciones de las ciencias humanas, sino que también surge de las ciencias naturales, renovadas y agrupadas en los campos de la cosmología, las ciencias de la Tierra y la ecología. ${ }^{17}$ Introducir este enfoque holístico, propio de los Estudios Generales, organiza un conocimiento antes disperso y compartimentado, como afirma este pensador.

Una educación universitaria que priorice las humanidades se convierte en una gran contribución a la acción educativa de nuestro tiempo. Para Morin, "la cultura de las humanidades [y las artes, agregaríamos nosotros] fue, sigue siendo y tiene que convertirse en una preparación

${ }^{15}$ J. Rodríguez Beruff, "Notas sobre la Educación General y la Facultad de Estudios Generales en la coyuntura de la certificación 46 del Senado Académico", Material fotocopiado sin datos de fecha, p. 13.

${ }^{16}$ C. Prieto, "Educación general, universidad y sociedad en el siglo 21", 2008, p. 4, en http://umbral.uprrp.edu/ponencia/educacion-general-universidad-y-sociedad-el-siglo-xix

${ }^{17}$ Morin, 2002, op. cit., p. 37. 
para la vida, pero no para una élite sino para todos". Según este intelectual, "literatura, poesía, cine, psicología tendrían que converger para volverse escuelas de comprensión humanas, una exigencia clave de nuestros tiempos de incomprensión generalizada". ${ }^{18}$

Estas propuestas constituyen un gran reto para las instituciones de educación superior dominicanas, que están caracterizadas por una excesiva profesionalización y por un ajuste desmedido a las demandas del mercado laboral. Por el contrario, una propuesta que no priorice la especialización temprana en el currículo universitario dominicano, y que responda a las necesidades sociales, aportaría grandes beneficios a los egresados que, cada cierto tiempo, tienen que reinventarse debido a los cambios de empleo y las bajas laborales que son usuales en esta época. Esta nueva visión de la enseñanza y el aprendizaje universitarios entronca con lo que hoy se conoce como "competencias génericas", tan demandadas por los mismos empleadores.

El texto de la Certificación 46 de la Facultad de Estudios Generales de la Universidad de Puerto Rico define la educación general como "un campo de experiencias y prácticas académicas coordinadas, constituido por el cruce de enfoques multi e interdisciplinares, elaborados a partir de grandes áreas del conocimiento y de la complejidad de los campos y las disciplinas emergentes". ${ }^{19}$ En un currículo tan determinado por las ocupaciones y las profesiones como el dominicano, este enfoque favorecería a que el estudiantado perciba que es posible progresar en las ciencias destruyendo, precisamente, lo que Morin llama "el aislamiento de las disciplinas".

Para finalizar, queremos resaltar que, a nuestro juicio, la mayor contribución de la educación general a los estudios universitarios de hoy, en el país y en Latinoamérica, sería aquella que nos permita dudar y reflexionar sobre esta exagerada profesionalización que caracteriza a la educación superior, sobre todo, a la dominicana. Como dice el matemático polaco Lichnerowicz: "tanto una gran parte de las actividades sociales, como el mismo desarrollo de la ciencia, necesita seres humanos

${ }^{18}$ Ibid., pp. 50 y 53 , respectivamente.

${ }^{19}$ Rodríguez Beruff, op. cit., p. 13. 
capaces de mantener un punto de vista más amplio para centrarse profundamente en los problemas y progresos nuevos que trasgredan las fronteras históricas de las disciplinas". ${ }^{20}$

No en vano, Morin afirma que no hay posibilidades simplistas o reduccionistas de situarse frente a esta nueva realidad de las sociedades humanas contemporáneas [... La única forma es abordarlas a partir de un razonamiento complejo que nos permita, al mismo tiempo, hablar de complementariedad, articulaciones y disociaciones. Por eso, hoy en día resulta más relevante, atractivo y significativo abordar temáticas generales que requieren múltiples visiones, conocimientos y técnicas para producir verdaderas alternativas sociales.

${ }^{20}$ Citado por Morin, 2002, op. cit., p. 13. 